SHORT REPORT

\title{
The diagnosis of hepatosplenic candidiasis by DNA analysis of tissue biopsy and serum
}

\section{A Kirby, C Chapman, I Hassan, J Burnie}

J Clin Pathol 2004;57:764-765. doi: 10.1136/icp.2003.015347

Hepatosplenic candidiasis is traditionally diagnosed by blood culture, magnetic resonance imaging (MRI), and histological analysis. The limitations of these methods include: low sensitivity (blood culture) and failure to isolate the organism (MRI/histology). This report describes a case of hepatosplenic candidiasis diagnosed by simultaneous polymerase chain reaction (PCR) analysis of liver biopsy and serum. PCR of biopsy and/or serum may offer a sensitive and specific diagnostic test for hepatosplenic candidiasis. Candida species can be identified, which helps guide antifungal chemotherapy, an important aspect in this difficult to treat infection.

\footnotetext{
A
} 37 year old woman with acute myeloid leukaemia received chemotherapy in February 2001. While she was neutropenic she was empirically treated for four weeks with amphotericin B for a suspected fungal sepsis. In July 2002, while in remission, computed tomography to investigate abdominal pain showed multiple localising liver lesions, which upon review were thought to be consistent with hepatosplenic candidiasis. A liver biopsy was sent for histological assessment, and appearances were "consistent with healing after a previous infarct, or possibly an old abscess". To investigate whether hepatosplenic candidiasis was the cause of the liver lesions we analysed serum and formalin fixed biopsy samples for candida DNA using the polymerase chain reaction (PCR).

\section{DNA ANALYSIS METHOD}

DNA was extracted from liver biopsy and serum using the QIAamp DNA mini kit (Qiagen, Hilden, Germany; tissue/ blood protocol as recommended by the manufacturer). The DNA was eluted in $200 \mu \mathrm{l}$ of molecular grade water and stored at $-20^{\circ} \mathrm{C}$.

The fungal internal transcribed spacer (ITS) region was amplified using universal fungal primers ITS86 and ITS4. ${ }^{1}$ The PCR mixture consisted of $25 \mu \mathrm{l}$ of IQ SYBR green supermix (Biorad, Hemel Hempstead, Hertfordshire, UK), $2 \mu \mathrm{l}$ of each primer $(50 \mathrm{pmol}), 10 \mu \mathrm{l}$ of template DNA, and molecular grade water to a final volume of $50 \mu \mathrm{l}$.

Icycler (Biorad) thermocycling conditions were: an initial denaturation of $95^{\circ} \mathrm{C}$ for three minutes, followed by 40 cycles of $95^{\circ} \mathrm{C}$ for 15 seconds and $62^{\circ} \mathrm{C}$ for 45 seconds. After amplification, a melt curve was performed using a cycling profile of $95^{\circ} \mathrm{C}$ for three minutes and $55^{\circ} \mathrm{C}$ for three minutes, followed by ramping of $1^{\circ}$ every 10 seconds to $94^{\circ} \mathrm{C}$. The PCR analysis also included both positive and negative controls.

To confirm species of candida, the PCR was repeated using species specific primers and Taqman probes. ${ }^{2}$ The following primers were selected because these species corresponded with the melt temperature (Tm) obtained.
- Candida parapsilosis: forward primer (5'-GGGTTTGG TGTTGAGCGATAC- $\left.3^{\prime}\right)$, reverse primer (5'-GGAGTTT GTACCAATGAGTGGAAA-3'), and Fam labelled probe (5'-CTCCGCCTTTCTTTCAAGCAAACCCAG-3').

- Candida tropicalis: forward primer (5'-CGTGGTAA CTTATTTTAAGCG-3'), reverse primer (5'-GCTTAAGTT CAGCGGGTAGTCCTA-3'), and Fam labelled probe (5'TGGCCACCATTTATTTCATAACTTTGACC-3').

- Candida glabrata: forward primer (5'-TTTCTCCTG CCTGCGCTTAA-3'), reverse primer (5'-ACGCACACT CCCAGGTCTTT-3'), and FAM labelled probe (5'AGAACAACCCACCAACCGCGCA-3').

The PCR mixture consisted of $25 \mu$ l of IQ supermix (Biorad), $2 \mu \mathrm{l}$ of each primer $(50 \mathrm{pmol}), 2 \mu \mathrm{l}$ of probe $(20 \mathrm{pmol}), 10 \mu \mathrm{l}$ of template DNA, and molecular grade water to a final volume of $50 \mu \mathrm{l}$.

\section{RESULTS}

The PCR was positive for candida DNA in both the sera and liver biopsy with fungal universal primers ITS86 and ITS4. The product gave a melt temperature of $89^{\circ} \mathrm{C}$. This melt profile corresponded to candida controls $C$ parapsilosis, $C$ tropicalis, and $C$ glabrata. The PCR was repeated using specific primers and FAM labelled probes, and only the $C$ parapsilosis primer set gave a positive PCR result.

\section{DISCUSSION}

DNA based diagnosis has the potential to diagnose all infectious diseases. As species specific DNA sequences are identified and diagnostic tests refined they will become more commonly used in diagnosis. The method described here had the advantage that it allowed the sample to be screened initially for fungal pathogens by using universal fungal primers and obtaining a Tm. The Tm was obtained with the use of SYBR green, which binds to double stranded DNA and fluoresces 50-100 times brighter than when not bound to DNA. At temperatures below the Tm of the product, SYBR green binds to the DNA and fluoresces brightly. As the Tm is reached, the DNA denatures and releases SYBR green, resulting in a decline in fluorescence. This decrease was plotted as fluorescence versus temperature. Plotting the negative first derivative of this data versus temperature change results in a melting peak and Tm for each product. The $\mathrm{Tm}$ is dependant on both the length of the amplified DNA and the G/C content of the sequence, factors that affect the energy needed to break the bonds in the DNA. The Tm can be compared with control samples. The Tm in this case matched the control samples of $C$ parapsilosis, $C$ tropicalis, and $C$ glabrata. Therefore, primers specific for these three species were selected and used in a repeat PCR analysis.

Abbreviations: ITS, internal transcribed spacer; MIC, minimum inhibitory concentration; PCR, polymerase chain reaction; Tm, melting temperature 


\section{Take home messages}

- We describe a case of hepatosplenic candidiasis diagnosed by polymerase chain reaction (PCR) analysis of both liver biopsy and serum

- Analysis of biopsy samples is likely to have a higher sensitivity than serum analysis

- PCR of biopsy and/or serum may offer a sensitive and specific diagnostic test for hepatosplenic candidiasis, and is particularly useful when culture is uninformative or the organisms are present in very low numbers

- In this case, the candida species was identified, which helps guide antifungal chemotherapy, an important aspect in this difficult to treat infection

We showed that the diagnosis of hepatosplenic candidiasis, and the identification of the infecting species, can be made by PCR analysis of histological samples and serum. PCR analysis was a useful diagnostic test in this situation because it allowed the diagnosis to be made even when there were low numbers of organisms (in the blood) and when the organisms were non-viable and could not be cultured (in the formalin fixed sample).

PCR analysis in hepatosplenic candidiasis may prove to be both a sensitive and specific test, although this needs to be confirmed in a series of patients. It also identifies the infecting species, information that is valuable in the selection of an antifungal. DNA serum analysis has been assessed before for disseminated candida infection, including hepatosplenic candidiasis, by Morace et al. ${ }^{3}$ PCR was used to investigate febrile neutropenic patients for disseminated candida infection, including hepatosplenic candidiasis. These authors found that 31 of their 72 patients were PCR positive. Thirteen of these patients were diagnosed as having disseminated candidiasis. This study showed that serum analysis offered a sensitive but non-specific diagnosis of disseminated candida infection. To avoid the need for an invasive (biopsy) sample, multiple samples were used to increase specificity for the identification of an infective pathogen. The testing gave a high negative predictive value $(97.5 \%)$ for the development of disseminated candidiasis in neutropenic patients. DNA analysis of biopsy samples may have a greater sensitivity than isolated serum analysis. This is based upon the notion that the amount of DNA at the source of infection would be greater than that found at a distant site (for example, in the blood). This is supported by the signal seen in the biopsy sample, which was far higher ( $>10$ times) than that found in the serum sample. Biopsy samples in combination with positive serum samples may also increase specificity.

\section{"Polymerase chain reaction was useful in our patient because a diagnosis was made and the candida species was identified"}

The diagnosis of hepatosplenic candidiasis is important because the infection causes severe morbidity and prolonged antifungal treatment is needed to obtain a cure. In one series, ${ }^{4}$ a median duration of 112 days of treatment was required. Because the treatment is protracted it is important to obtain guidance on which antifungal to use. Knowledge of the species of candida that is infecting can help guide treatment because candida shows species specific antifungal resistance patterns..$^{5-8}$ Candida glabrata and C krusei are fluconazole resistant and $C$ guilliermondii is amphotericin resistant. The minimum inhibitory concentration (MIC) of caspofungin/micafungin is raised with respect to $C$ parapsilosis and the MIC of flucytosine is raised with respect to $C$ tropicalis and $C$ krusei.

PCR was useful in our patient because a diagnosis was made and the candida species was identified. The organism was identified as $C$ parapsilosis. Therefore the patient was rationally started on oral azole treatment, which enabled her to be discharged home. It also allowed us to avoid treatment with caspofungin. This may be appropriate because some reports have shown persistent $C$ parapsilosis candidaemia on caspofungin. ${ }^{9} 10$

The patient was probably infected by $C$ parapsilosis during her fungal sepsis the year before, despite no pathogen being identified, with the long latent period being caused by partial treatment with amphotericin B. This case highlights the importance of taking factors that promote fungal infection seriously-for example, broad spectrum antibiotics-and of responding appropriately to evidence of an avoidable risk of infection-for example, colonisation of an intravenous catheter with candida should lead to its removal.

In conclusion, we have shown that hepatosplenic candidiasis can be diagnosed by DNA analysis of biopsy samples, potentially with a higher sensitivity than serum analysis. Therefore, DNA analysis of biopsy/serum samples may help in the diagnosis of hepatosplenic candidiasis when hepatosplenic candidiasis is clinically considered but insufficient evidence for diagnosis is available from traditional diagnostic methods, or where the diagnosis is made but no infecting pathogen has been identified. Identification of the infective pathogen may help guide antifungal chemotherapy; this would be of particular benefit in patients where correct treatment is important-for example, patients refractory to their empirical treatment or patients where further chemotherapy (and therefore neutropenia) is planned. Authors are only allowed one affiliation each.

\section{Authors' affiliations}

A Kirby, I Hassan, Department of Medical Microbiology, Clinical Sciences Building, Manchester Royal Infirmary

C Chapman, J Burnie, NeuTec Pharma plc, Clinical Sciences Building, Manchester Royal Infirmary

Correspondence to: Dr A Kirby, Department of Medical Microbiology, Manchester Royal Infirmary, Oxford Road, Manchester M13 9WL, UK; kirbyandrew@doctors.org.uk

Accepted for publication 31 January 2004

\section{REFERENCES}

1 Turenne CY, Sanche SE, Hoban DJ, et al. Rapid identification of fungi using the ITS2 genetic region and an automated fluorescent capillary electrophoresis system. J Clin Microbiol 1999;37:1846-51.

2 Guiver M, Levi K, Oppenheim BA. Rapid identification of candida species by TaqMan PCR. J Clin Pathol 2001;54:362-6.

3 Morace G, Pagano L, Sanguinetti $M$, et al. PCR-restriction enzyme analysis for detection of candida DNA in blood from febrile patients with haematological malignancies. J Clin Microbiol 1999;37:1871-5.

4 Sallah S, Semelka RC, Wehbie R, et al. Hepatosplenic candidiasis in patients with acute leukaemia. Br J Haematol 1999; 106:697-701.

5 Marco F, Danes C, Almela M, et al. Trends in frequency and in vitro susceptibilities to antifungal agents, including voriconazole and anidulafungin, of candida bloodstream isolates. Results from a six-year study (1996-2001). Diagn Microbiol Infect Dis 2003;46:259-64.

6 Barchiesi F, Schimizzi AM, Fothergill AW, et al. In vitro activity of the new echinocandin antifungal, MK-0991, against common and uncommon clinical isolates of candida species. Eur J Clin Microbiol Infect Dis 1999;18:302-4.

7 Pfaller MA, Diekema DJ, Messer SA, et al. International Fungal Surveillance Participant Group. In vitro activities of voriconazole, posaconazole, and four licensed systemic antifungal agents against candida species infrequently isolated from blood. J Clin Microbiol 2003;41:78-83.

8 White CT, Marr KA, Bowden RA. Clinical, cellular, and molecular factors that contribute to antifungal drug resistance. Clin Microbiol 1998;1 1:382-402.

9 Mora-Duarte J, Betts R, Rotstein C, et al. Comparison of caspofungin and amphotericin B for invasive candidiasis. N Engl J Med 2002;347):2020-9.

10 Walsh TJ. Echinocandins - an advance in the primary treatment of invasive candidiasis. N Engl J Med 2002;347:2070-2. 